\title{
Partial Discharge Modelling Based on a Cylindrical Model in Solid Dielectrics
}

\author{
George Chen and Fauzan Baharudin \\ School of Electronics and Computer Science \\ University of Southampton, United Kingdom \\ Email: gc@ecs.soton.ac.uk
}

\begin{abstract}
Partial discharges (PDs) are harmful to electrical insulation as they may degrade the material. Consequently, a lot of efforts have been made to develop suitable systems for PD measurements and monitoring. Due to the complexity of PDs and many factors that can influence PD characteristics, the research on PDs is still very active and several models have been proposed over the years to understand the behaviours of PDs.

In the present paper, we have investigated the frequency characteristics of a cylindrical void in solid dielectric based on the model proposed by Forssen. Using similar program environments of MATLAB and COMSOL, our algorithm performs better in computation time and gives the similar results compared with the experiments. More importantly, a new interface has been developed so users are able to change parameters. This new feature allows the user to examine effects of various factors such as conductivity of the cavity surface, the applied voltage and statistical time lag on PD characteristics.
\end{abstract}

Index Term--Partial discharge, simulation, solid dielectrics, frequency effect.

\section{INTRODUCTION}

$\mathrm{P}$ artial discharges (PD) are localised electric discharges that do not bridge the whole distance between electrodes; indicating the presence of cavity and defects in the insulation such as high voltage power cables and power generators. The measurement and investigation of partial discharges in cavity to diagnose the condition of the insulation system has been of interest since the early 1940's. PDs are generally divided into three different groups because of their different origins: (1) corona discharges, (2) internal discharges and (3) surface discharges [1].

Traditionally, PDs are only measured and examined at a single frequency of the applied voltage. However, it is now possible to measure partial discharges at variable frequencies of the applied voltage using a rather new technique called Variable Frequency - Phase Resolved PD Analysis (VFPRPDA) method [2]. The new technique provides researchers more information and data about the state of the insulation system as compared to a single frequency.

In the present paper, we have investigated the frequency characteristics of a cylindrical void in solid dielectric based on the model proposed by Forssen [3]. Using similar program environments of MATLAB and COMSOL, a new interface has been developed so users are able to change parameters. This new feature allows the user to examine effects of various factors such as conductivity of the cavity surface, the applied voltage and statistical time lag on PD characteristics.

\section{PD MODEL}

In laboratory investigation a sandwich model is often used as shown in Figure 1. For comparison we use the same arrangement as in [3]. Three polycarbonate plates each of 1 $\mathrm{mm}$ thick and $14 \mathrm{~mm}$ in diameter are placed on top of each other with the middle plate being drilled at the centre to resemble a void with $10 \mathrm{~mm}$ of diameter. A $10 \mathrm{kV}$ sinusoidal voltage was initially applied between the electrodes to investigate the influence of frequency, ranging from $0.01 \mathrm{~Hz}$ to $100 \mathrm{~Hz}$. To prevent corona discharges the sample and electrodes are typically casted with epoxy resin.

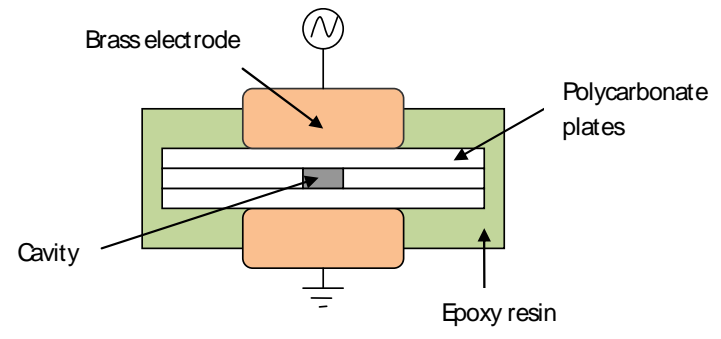

Figure 1 Illustration of the PD model simulated.

The basic governing equations are as below:

$$
\begin{gathered}
\nabla \cdot D=\rho_{f} \\
\nabla \cdot J_{f}+\frac{\partial \rho_{f}}{\partial t}=0
\end{gathered}
$$

with $\mathrm{D}$ as the electric displacement field, $\rho_{f}$ as the free charge density, and $\mathrm{J}_{\mathrm{f}}$ as the free current density. But for nondispersive, linear isotropic dielectrics exposed to slowly varying fields, equations 1 and 2 can be combined to obtain equation 3 ; where $\sigma$ equals to the electric conductivity, $\mathrm{V}$ equals to the applied electric potential and $\varepsilon$ as the permittivity. 


$$
\nabla \cdot\left(-\sigma \nabla V-\varepsilon \nabla\left(\frac{\partial V}{\partial t}\right)\right)=0
$$

Partial discharges will occur if and only if the insulation systems satisfy two necessary conditions: (1) There must be free electrons available at the surfaces of the void to start an electron avalanche process and (2) The electric field must be high enough, corresponding to the associated voltage called the Inception Voltage, $\mathrm{U}_{\text {inc }}$.

\section{A. Statistical Time Lag}

The period between $U_{\text {inc }}$ and the starting point of the electron avalanche due to the delay in getting free electrons is called Statistical Time Lag, $\boldsymbol{\tau}_{\text {stat }}$. The discharges will then continue until the electric field in the cavity becomes too low such that it reaches a specific voltage called the Extinction Voltage, $\mathrm{U}_{\text {ext }}$.

If $\tau_{\text {stat }}$ is negligible compared to the period of the applied voltage, $T_{v}$, it will not be included in the simulation to speed up processing. Up until now however, there is no clear distinction between significant and negligible $\boldsymbol{\tau}_{\text {stat }}$ value and this imposes problems when modelling PD activity. Therefore, the following criteria have been introduced in this simulation to determine the borderline between significant and negligible value for PD analysis as below:

$$
\begin{array}{ll}
\frac{T_{v}}{\tau_{\text {stat }}} \geq 10000 & \text { Negligible } \\
\frac{T_{v}}{\tau_{\text {stat }}}<10000 & \text { Significant }
\end{array}
$$

\section{B. Frequency dependent nature of PDs}

There are also two time constants that affect and influence the PD activity: (1) Charge redistribution time on the cavity surface, $\tau_{\text {cavity. }}$. Determined from the conductivity of the cavity surface, $\sigma_{\text {surf }}$, and the geometry of the void; e.g. a higher $\sigma_{\text {surf }}$ and/or a smaller void will results in a shorter $\tau_{\text {cavity. }}$. (2) The charge redistribution time in the surrounding insulation material, $\tau_{\text {material. }}$. Determined from the conductivity and permittivity of the bulk (surrounding) insulation material as well as its geometry; e.g. an increase in conductivity and/or a decrease in permittivity will results in a shorter $\tau_{\text {material }}$.

PD activity is dependent on the frequency of applied voltage only when:

$$
\left[\tau_{\text {cative }} \text { and / or } \tau_{\text {material }}\right]<\text { or } \ni T_{v} \text { and / or ; }
$$$$
\left[\tau_{\text {stat }}\right]>\text { or э } T_{v}
$$

where $\ni$ means "within the same range as". Both $\tau_{\text {cavity }}$ and $\tau_{\text {material }}$ value can be associated with the 'Screening Effect' and 'Blocking Effect' depending on mutual relationship between them.

PD activity in the cavity was modelled as an increase in the associated channel conductivity value and they were modelled to behave as equation 6 below; where $\sigma_{0}$ is the initial channel's electrical conductivity value before discharging, I is the current across the channel, $U$ is the electric potential difference across the channel and $I_{\text {crit }}$ is the critical current value to start an electron avalanche [3]. For numerical reasons the conductivity needs to be limited to a maximum value of $\sigma_{\max }\left(\right.$ i.e. $1 \times 10^{-4}$ ) in order to achieve numerical convergence for the final solution and calculation.

$$
\begin{gathered}
\sigma= \begin{cases}\sigma_{0} e^{\left(\left|\frac{v}{v_{\text {int }}}\right|+\mid \frac{I}{I_{\text {crit }}}\right)} & \text { during disch } \arg e \\
\sigma_{0} & \text { otherwise }\end{cases} \\
I= \begin{cases}\iint_{S} J d S=\iint_{S} \sigma \nabla V d S & \text { during discharge } \\
0 & \text { otherwise }\end{cases}
\end{gathered}
$$

Equation 7 above is the equation for the current through the discharging channel [3]. Notice that $I$ and $\sigma$ in both of the equations above are dependent on each other. The only difference is that they are of a different time step; i.e. $\sigma$ takes the value of I from the previous time step.

At the frequency where $\tau_{\text {stat }}$ is significant in comparison to the $T_{v}$, equation 8 below needs to be used in addition to the $\mathrm{U}_{\text {inc }}$ limit alone to initiate PD [3]:

$$
P= \begin{cases}\frac{I_{e 00} e^{\left|\frac{U}{U_{i n c}}\right|}}{N} d t & U>U_{i n c} \\ & \\ 0 & U<U_{i n c}\end{cases}
$$

where $\mathrm{P}$ is the probability of PD occurring, $\mathrm{I}_{\mathrm{e} 0}$ is the initial electron injection intensity and $\mathrm{N}$ is the number of channels inside the cavity. The value of $\boldsymbol{d} t$ needs to be calculated using equation 9:

$$
d t=\frac{\text { time stepping int erval during no PD activity }}{360^{\circ} \times \text { frequency }}
$$

so that $\mathrm{P}$ can be compared with a random number $\mathrm{R}$ which lies within the range of $0<\mathrm{R}<1$ such that [3]:

$$
\begin{array}{ll}
P>R & \text { PD occurs } \\
P<R & \text { no PD occurs }
\end{array}
$$

For the case of having more than one channel that exceeds $\mathrm{U}_{\mathrm{inc}}$, only one channel will be chosen randomly to discharge by MATLAB.

\section{COMSOL and MATLAb in Brevity}

The COMSOL software used in this project based entirely on the finite element method (FEM) structure analysis. It 
solves various physical problems by finding the approximate solution of partial differential equations (PDEs) and then renders them into equivalent ordinary differential equations (ODEs) to be solved using standard techniques such as the Newton-Raphson iteration method [4]. The full equation used by COMSOL in solving the electromagnetics module is as below:

$$
e_{a} \frac{\partial^{2} V}{\partial t^{2}}+d_{a} \frac{\partial V}{\partial t}+\nabla \cdot(-c \nabla V-a V+\gamma)+a V+\beta \nabla V=f
$$

When the entire zero valued variable from Table are eliminated, the resulting equation is simplified and becomes:

$$
-\nabla \cdot d\left(\sigma+j w \varepsilon_{0} \varepsilon_{r}\right) \nabla V=0
$$

where $d$ is the thickness of the sample, $\omega$ is the frequency in radians and $\varepsilon_{0}$ as the permittivity of vacuum and $\varepsilon_{r}$ as the relative permittivity.

Table I

Variable definitions for equation 11

\begin{tabular}{|c|c|c|c|c|}
\hline $\mathrm{a}$ & $=$ & Absorption coefficient & $=$ & 0 \\
\hline $\mathrm{f}$ & $=$ & Source term & $=$ & 0 \\
\hline $\mathrm{e}_{\mathrm{a}}$ & $=$ & Mass coefficient & $=$ & 0 \\
\hline $\mathrm{d}_{\mathrm{a}}$ & $=$ & Damping coefficient & $=$ & 0 \\
\hline$\alpha$ & $=$ & Conservative flux convection & $=$ & $0 \mid 0$ (matrix) \\
& & coefficient & & \\
\hline$\beta$ & $=$ & Convection coefficient & $=$ & $0 \mid 0$ (matrix) \\
\hline$\gamma$ & $=$ & Conservative flux source term & $=$ & $0 \mid 0$ (matrix) \\
\hline $\mathrm{c}$ & $=$ & Diffusion coefficient & $=$ & $\mathrm{d} .\left(\sigma+\mathrm{j} \omega \varepsilon_{0} \varepsilon_{\mathrm{r}}\right)$ \\
\hline
\end{tabular}

MATLAB on the other hand combines both numerical computing environment and programming language. Using matrix manipulations, MATLAB allows the user to easily plot functions or data, implement algorithms, and create graphical user interfaces (GUI). With seamless interoperability between COMSOL and MATLAB, they have been widely used by researchers and designers to model, simulate and solve various physical problems in the engineering field.

The model was drawn under the 2D axial symmetry space dimension using the AC/DC module within the COMSOL. To add some random behaviour of the PD activity, the void was divided into 5 different channels; each having equal volume of space. This was done to illustrate that the discharges may occur at only some parts of the cavity. Cavity surface was added in the model by adding layers of $0.1 \mathrm{~mm}$ thick surrounding the void.

\section{COMSOL with MATLAB Interface}

Iterative elements were introduced in the model using MATLAB. The coding was structured initially using low frequency value of the applied voltage and then followed by a higher frequency; mainly because of the $\boldsymbol{\tau}_{\text {stat }}$ factor. COMSOL provides an excellent interoperability with MATLAB.

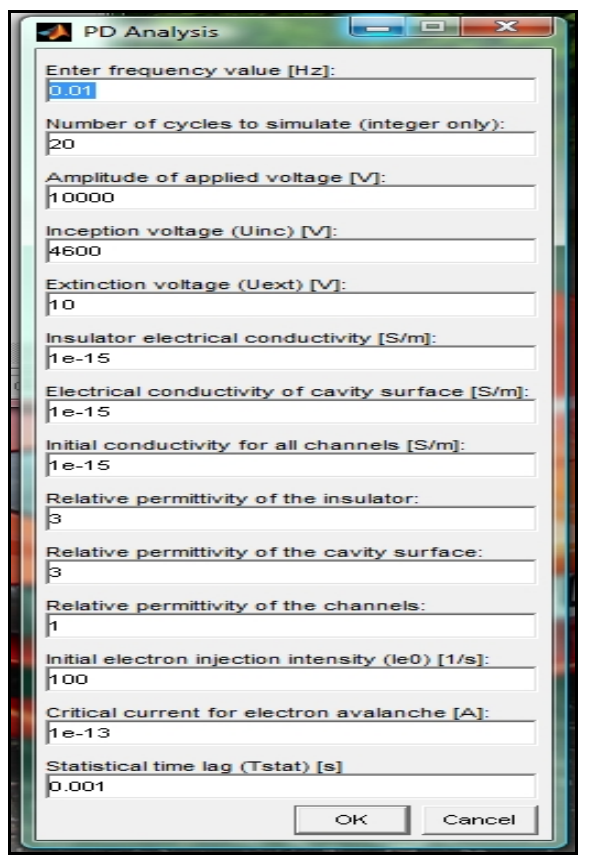

Figure 2 Interactive menu at the beginning of the simulation with default parameter values

Initial analysis revealed that running 20 cycles is sufficient to achieve a respectable result and feasible simulation time. To increase simulation speed further, the variables were preallocated initially using the 'zeros' function wherever possible; especially within a loop (the coding itself contains more than 20 variable names). In addition, the whole coding structure was made on the basis of having minimum conditional syntaxes and iterations where possible.

To investigate the effect of the amplitude the applied voltage the voltage was varied from $8 \mathrm{kV}$ to $12 \mathrm{kV}$ in $2 \mathrm{kV}$ steps to see any effects towards the PD activity inside the channels. The $\sigma_{\text {surf }}$ was also varied from $1 \times 10^{-10}$ up to $1 \times 10^{-20} \mathrm{~S} / \mathrm{m}$ to see any variations in the PD activity. Most of the additional investigations were simulated once at the low frequency region and once again at the high frequency. With the help of interactive menu at the start of the simulation as shown in Figure 2, the variables used for the additional investigations can be keyed in easily and quickly.

\section{RESULTS AND DISCUSSIONS}

\section{A. Average PD per Cycle}

The $0.1 \mathrm{~Hz}$ frequency took the longest time of approximately 16 hours to finish simulating the model whereas the $100 \mathrm{~Hz}$ took the shortest duration of 11 hours. It might be safe to assume generally that the time it takes to simulate the model for each frequency is inversely proportionate with the frequency of the applied voltage. However, it does not necessarily means that the PD activity will also follow this trend and this is reflected in the $0.01 \mathrm{~Hz}$ data where the duration is in fact shorter than the next higher 
frequency which is the $0.1 \mathrm{~Hz}$. To explain this further, refer to Figure 3.

At $0.01 \mathrm{~Hz}$, its average PD per cycle is in fact lower than the average PD per cycle of the $0.1 \mathrm{~Hz}$ frequency. This means, the total simulation time per frequency is behaving proportionally to the number of discharges per cycle; and this seems to be logical because for every PD occurrences, MATLAB will switch into the PD activity program loop which uses $0.02^{\circ}$ as the time stepping interval. For example, instead of stepping from $45^{\circ}$ to $48^{\circ}$ (default $3^{\circ}$ interval), MATLAB will sweep in $0.02^{\circ}$ time step from $45^{\circ}$ up to an angle at which the electric potential across the specific discharging channel reaches $\mathrm{U}_{\text {ext }}$ (usually after 3 times of $0.02^{\circ}$ time step i.e. $45.06^{\circ}$ ). Only then MATLAB will revert back to the $3^{\circ}$ time steps and repeat the simulation again starting at $48^{\circ}$ phase angle. In other words, COMSOL will be called at least three times more to account for the additional time stepping interval.

At frequencies above $0.1 \mathrm{~Hz}$ (i.e. where the $\tau_{\text {stat }}$ is significant), the program will simulate $\tau_{\text {stat }}$ influence to the PD activity by including equation 8,9 and 10 in choosing only one to discharge for the next time step. Clearly, the $1 \mathrm{~Hz}$ frequency is affected by the inclusion of those additional equations and hence extra time spent. Although the additional time might not be much, but over 20 cycles, the time delay sums up to cost $1 \mathrm{~Hz}$ frequency additional 30 minutes; longer than $0.01 \mathrm{~Hz}$ even though $0.01 \mathrm{~Hz}$ has more PD.

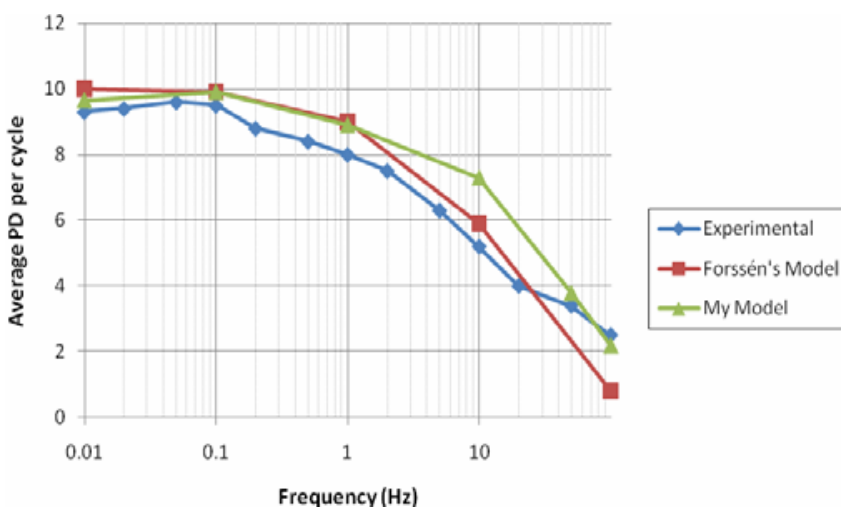

Figure 3 Average PD per cycle for various frequencies: lab experiment, existing PD simulation model and present simulation.

Referring to the trend line in Figure 3, it can be seen that close agreements have been reached between the simulated model, Forssén's model and the actual practical experiment [3]. This ultimately proves that the simulation model produced and programming code generated for this project is correct and acceptable as a whole to be used for any other PD analysis using different parameter values.

\section{B. $\tau_{\text {cavity }}$ and $\tau_{\text {material }}$ Influences}

Altering $\sigma_{\text {surf }}$ value is one way of investigating the relationship between $\tau_{\text {cavity }}$ and $\tau_{\text {material }}$ and their influences towards PD activity because $\tau_{\text {cavity }}$ behaves inversely proportional to $\sigma_{\text {surf }}$. Simulations were done for $\sigma_{\text {surf }}$ using values of $1 \times 10^{-10} \mathrm{~S} / \mathrm{m}, 1 \times 10^{-15} \mathrm{~S} / \mathrm{m}$ (default) and $1 \times 10^{-20} \mathrm{~S} / \mathrm{m}$ at $0.1 \mathrm{~Hz}$ and $50 \mathrm{~Hz}$ of the applied frequency. The insulation conductivity value, $\sigma_{\text {ins }}$, was fixed at $1 \times 10^{-15} \mathrm{~S} / \mathrm{m}$ throughout all simulations. Table 2 summarises the simulated results for $0.1 \mathrm{~Hz}$ and $50 \mathrm{~Hz}$.

Table II

Average number of PD per cycle using various cavity surface conductivity values; simulated at $0.1 \mathrm{~Hz}$ and $50 \mathrm{~Hz}$ frequency.

\begin{tabular}{|c|c|c|c|}
\hline \multirow{2}{*}{$\begin{array}{c}\text { Applied } \\
\text { frequency (Hz) }\end{array}$} & \multicolumn{3}{|c|}{ Average PD per cycle } \\
\cline { 2 - 4 } & $\mathbf{1 \times 1 0 ^ { - 2 0 }} \mathbf{S} / \mathbf{m}$ & $\begin{array}{c}\mathbf{1 \times 1 0 ^ { - 1 5 }} \mathbf{S} / \mathbf{m} \\
(\mathbf{d e f a u l t})\end{array}$ & $\mathbf{1 \times 1 0 ^ { - 1 0 }} \mathbf{S} / \mathbf{m}$ \\
\hline $\mathbf{0 . 1}$ & 10.05 & 9.9 & 9.9 \\
\hline $\mathbf{5 0}$ & 4.4 & 3.8 & 3.6 \\
\hline
\end{tabular}

There will be no difference at all in terms of physical characteristics when the cavity surface and the surrounding insulation material are defined using the default values for their electrical conductivity and relative permittivity (refer to Figure 2); i.e. $\left(\tau_{\text {cavity }}=\tau_{\text {material }}\right)$. However, increasing $\sigma_{\text {surf }}$ to $1 \times 10^{-10} \mathrm{~S} / \mathrm{m}$ (to simulate ageing) will effectively decrease the value of $\tau_{\text {cavity; }}$, turning the relationship into $\left(\tau_{\text {cavity }}<\tau_{\text {material }}\right)$. This causes the 'Screening Effect' to occur which effectively reduces the average number of PD per cycle. The opposite is true for reducing $\sigma_{\text {surf }}$; causing the 'Blocking Effect' to occur as mentioned in the Background Reading section. Table confirms these relationships as it suggested that the average number of PD per cycle will decrease as the $\sigma_{\text {surf }}$ increases.

\section{Altering the Amplitude of the Applied Voltage}

At low amplitude e.g. $8000 \mathrm{~V}$, the average time it took for any of the channels to stay above the $\mathrm{U}_{\text {inc }}$ limit will be much less than the one experiencing high amplitude of the applied electric potentials e.g. $12000 \mathrm{~V}$. This directly decreases the chances for any of the channels to discharge and thus the average PD per cycle will reduce. Conversely is true for the high amplitude of the applied voltage (refer to Table 3).

Table III

The effect of altering the amplitude of the applied voltage; simulated at $50 \mathrm{~Hz}$

\begin{tabular}{|c|c|c|c|}
\cline { 2 - 4 } \multicolumn{1}{c|}{} & \multicolumn{3}{c|}{ Amplitude of the applied voltage (V) at $\mathbf{5 0 H z}$} \\
\cline { 2 - 4 } \multicolumn{1}{c|}{} & $\mathbf{8 0 0 0 0}$ & $\mathbf{1 0 0 0 0}$ & $\mathbf{1 2 0 0 0}$ \\
\hline $\begin{array}{c}\text { Average PD per } \\
\text { cycle }\end{array}$ & 1.75 & 3.8 & 5.45 \\
\hline
\end{tabular}

\section{CONCLUSIONS}

COMSOL and MATLAB work seamlessly with each other, making it possible to simulate the complex and random nature of PD activities. The simulation platform established in the present work is proven to be ideal for simulating PD activity in an otherwise homogeneous dielectric material exposed to high electric fields. This simulation platform is better than the existing model in the following two aspects: 
In the present simulation platform, the value $\boldsymbol{d t}$ changes as the frequency changes, making it versatile for all simulation work and more efficient.

The interactive menu introduced is useful for further investigations using various material properties or $\mathrm{PD}$ parameter values.

Specifically for the PD activity, it can be summarised that the $\tau_{\text {stat }}$ value affects the number of average PD per cycle, at the frequency above $0.1 \mathrm{~Hz}$ i.e. where the $\tau_{\text {stat }}$ is found be significant to the $T_{v}$, determined using equation 4 .

Varying the $\sigma_{\text {surf }}$ value will in effect alter the value of $\tau_{\text {cavity }}$ and in turn observes the 'Screening Effect' or the 'Blocking Effect'. Screening occurs when $\left(\tau_{\text {cavity }}<\tau_{\text {material }}\right)$ and blocking occurs when $\left(\tau_{\text {cavity }}>\tau_{\text {material }}\right)$.

Lastly, lowering the amplitude of the applied voltage will reduce the average number of PD per cycle due to the reduced area above the $\mathrm{U}_{\text {inc }}$ line and vice-versa.

\section{REFERENCES}

[1] F. H. Kreuger, Discharge Detection in High Voltage Equipment, Butterworths, London, 1989.

[2] Hans Edin, Partial Discharges Studied with Variable Frequency of the Applied Voltage. Sept 14, Stockholm, Sweden: Kungl Tekniska Högskolan, Vols. TRITA-EEK-0102. ISSN 1100-1593, 2001.

[3] H. Edin and C. Forssen. Modelling of a discharging cavity in a dielectric material exposed to high electric fields. KTH IR-EE-EEK. 2005, 2005:003

[4] J. H. Mathews and K. D. Fink, Numerical Methods: Using Matlab, Fourth Edition, Prentice- Hall Pub. Inc, 2004. 